\title{
Twins prematurity - the influence of prenatal surveillance
}

\author{
Ana Patrícia Domingues ${ }^{1}$, Etelvina Fonseca ${ }^{1}$, Adriana Belo ${ }^{2}$, and Paulo Moura ${ }^{1,3}$ \\ 'Obstetrics A Department, Maternidade Dr Daniel de Matos, Centro Hospitalar e Universitário de Coimbra, Portugal, ${ }^{2}$ Biostatistics MSc, Coimbra, \\ Portugal, and ${ }^{3}$ Obstetrical Clinic of the Faculty of Medicine, Coimbra University, Portugal
}

\begin{abstract}
Objective: To evaluate the influence of the local prenatal surveillance of twin pregnancies in the obstetrical results.

Methods: A prospective cohort study of multiple pregnancies delivered over a period of 16 years in a tertiary centre was conducted. In this study 861 twin pregnancies were included. They were compared for obstetric complications, gestational age at delivery, mode of delivery and birthweight, according to the place of the surveillance.

Results: Of the 861 cases examined, the following obstetric complications were significantly different: metrorrhagia $(p=0.039)$, infections $(p<0.001), \operatorname{HELLP}(p=0.007)$, PROMPT $(p<0.001)$ and fetal death $(p=0.024)$. The mode of delivery was similar but occurred mostly $\leq 32$ weeks $(p<0.001)$, the birthweight was mostly $<2000 \mathrm{~g}$ and occurred more NICU admission $(p<0.001)$ when surveillance was outside the MPC-MDM.

Conclusion: Our results demonstrate the crucial importance of prenatal surveillance be carried in a differentiated referral centers with specific/strict protocols or the urgent implementation of same protocols in all other places of surveillance, since this straight surveillance greatly reduces the occurrence of prenatal complications, mainly PROMPT, PTD.
\end{abstract}

Keywords

Reducing twins prematurity, twins obstetrical complications, twins prenatal surveillance

\section{History}

Received 23 July 2013

Revised 7 October 2013

Accepted 8 July 2014

Published online 31 July 2014

\section{Introduction}

Since 1980 there has been a worldwide increase in multiple births. This seems to be due to an increase in the age of reproduction, the use of ovulation induction (OI) and the use of assisted reproduction techniques such in vitro fertilization (IVF) and intracytoplasmatic sperm injection (ICSI). It is well-known that maternal and obstetrical complications are more frequent in twin pregnancies than in singleton's [1-6]. However, there is no specific information regarding the pregnancy outcomes according to the place/protocols of surveillance. This is a major important issue due to the subjective impression and secondary conclusions of some studies, that surveillance of twin pregnancies in referral centers which generally have strict protocols lead to better outcomes. Also it is important to establish the limits of obstetrical surveillance between all health professionals.

The aim of the present study was to evaluate the influence of the local of prenatal surveillance of twin pregnancies in their obstetrical results.

Address for correspondence: Ana Patrícia Domingues, Obstetrics A Department, Maternidade Dr Daniel de Matos, Centro Hospitalar e Universitário de Coimbra, Rua Miguel Torga, 3030 - 165 Coimbra, Portugal. $\quad$ Tel: $\quad+351239403060$. anapatriciadomingues@hotmail.com

\section{Materials and methods}

Data from files of all twin pregnancies who deliver in our tertiary obstetric centre over a period of 16 years (between January 1996 and December 2011) were prospectively collected and analyzed in a cohort study. No intervention was done beside the normal twin surveillance protocol in those with surveillance in our center. This study was included in an investigation project approved by the Ethics Committee of the University Hospitals of Coimbra.

Twin pregnancies were all included (dichorionic and monochorionic) and higher order multiple pregnancies whose surveillance is unanimous to be done in a referral center were excluded.

The obstetric complications included were first trimester bleeding; urinary, genital or other infections; anemia; fetal malformations; hypertensive diseases of the pregnancy (gestational hypertension, preeclampsia, eclampsia and HELLP syndrome); gestational diabetes - GD; preterm delivery threat, preterm delivery - PPT, considered delivery occurred after 24 and before 37 weeks of gestation; preterm premature rupture of membranes - PPROM, considered the occurrence of premature rupture of membranes after 24 and before 37 weeks of gestation; intrauterine growth restriction - IUGR, defined as fetal measure of abdominal perimeter $<10$ th centile; discordant growth restriction, defined as $20 \%$ difference between fetal birthweight and fetal death. 
About birth, gestational age at delivery (categorized in the following classes: $<28,28-32,33-34,35-36$ and $>36$ weeks), mode of delivery (categorized in vaginal, cesarean during delivery, elective cesarean and cesarean to the second twin), birthweight of both twins and admission of the neonates in the Neonatal Intensive Care Unit (NICU) were analyzed.

Gestational age was calculated from the last menstrual period, confirmed and corrected by the time of the first trimester ultrasound. Chorionicity was also determined in the first trimester scan (by lambda and $\mathrm{T}$ signs).

We performed a global characterization of the sample of 861 twin pregnancies, considering the following parameters: complications during pregnancy, type of delivery, gestational age at delivery, postpartum complications, NICU admission and birthweight of each newborn. These parameters were characterized by absolute frequencies and relative frequencies.

Our sample was defined by five groups of women according to the place where they were monitored during pregnancy - in Multiple Pregnancy Consultation of our Unit (MPC-MDM), in any district hospital (DH), with their general practitioner (GP), in private doctors (PD) or without any kind of surveillance (WS). The care pathway followed by these twin pregnancies in DH, GP and PD was similar to a singleton pregnancy surveillance with an ultrasound and blood pregnancy routines once a trimester. The appointments were generally once every 4 weeks and when complications were detected or at delivery time they were directed towards the structure in charge of their delivery (MPC-MDM). The care pathway in MCP-MDM followed a strict protocol that included: general measures (night rest of 8-10 hours; short period rest after lunch; suspension of work activities by 22-24 weeks), first trimester (blood pregnancy 1st trimester routines, chorionicity determination before the 12nd week of gestation if possible and first trimester ultrasound at 12-13 weeks), second trimester (appointments every $4 / 4$ or $3 / 3$ weeks - depending on chorionicity, risk factors and presence of obstetric complications; ultrasound evaluation in every appointment with fetal echocardiography and cervical length at 20-24 weeks; blood pregnancy 2nd trimester routines; vaginal and cervical swab at 22-24 weeks) and third trimester surveillance (appointments every $3 / 3$ or $2 / 2$ weeks - depending on chorionicity, risk factors and presence of obstetric complications until 34th week and every week after that; ultrasound evaluation in every appointment until 34 week and individualized after that according to pregnancy evolution and complications; blood pregnancy routines of the 3rd trimester; streptococcus screening at 3234 weeks). Antenatal corticosteroids (betamethasone in two doses of $12 \mathrm{mg}$ each, given $24 \mathrm{~h}$ apart) were offered to women considered to be at risk of preterm delivery (spontaneous or iatrogenic) up to 34 weeks gestation. Tocolysis when necessary was attempted with atosiban. Delivery was programmed at 37 weeks in monochorionic and at 38 weeks at dichorionic twins. Elective cesarean section indications included first twin in a non cephalic presentation, second twin with a birthweight estimate inferior to $2000 \mathrm{~g}$, especially if in a non-cephalic presentation, twin-to-twin transfusion syndrome, maternal or fetal indication.

The differences between the proportions of the groups were evaluated using the chi-square test.
We then constructed two logistic regression models, one to determine the predictors of pregnancy complications and another to determine the predictors of gestational age at delivery less than 35 weeks. These models allowed us to evaluate whether the site of prenatal surveillance had significant influence on each of the outcomes mentioned.

The level of significance used in this analysis was 5\% and the statistical software used was SPSS ${ }^{\circledR}$ v19.0.0.2 (SPSS Inc., Chicago, IL).

\section{Results}

During the study period - January 1996 to December 2011 there were 919 deliveries of multiple pregnancies in our center. Of these, 861 twin pregnancies were included in our study. Nine women had no kind of surveillance (1\%), 485 were followed in MPC-MDM (56.3\%), 36 at the GP (4.2\%), 252 in PD (29.3\%) and 79 in other district hospitals (9.2\%).

The analysis of obstetrical complications - Table 1 concerning anemia, urinary track infections, preterm delivery threat, gestational hypertension, preeclampsia, gestational diabetes, fetal malformations, twin-to-twin transfusion syndrome in monochorials, intrauterine discordant growth and intrauterine growth restriction revealed no major statistical significant differences between the groups.

First trimester bleeding was higher in the women from DH and lower in those from PD. The prevalence of infections other then urinary was significantly higher in MPC-MDM. A higher rate of HELLP syndrome was verified in women from WS and DH. Preterm premature rupture of membranes was less frequent in the MPC-MDM and higher in DH.

Intrauterine fetal death was also significantly less frequent in MPC-MDM women and more frequent in those from GP and PD.

Regarding delivery, there were no significant differences in cesarean rates - Table 2.

Gestational age at delivery, birth weight and admissions to NICU were statistically different - Table 2 . There were

Table 1. Obstetrical complications occurrence according to place of prenatal surveillance.

\begin{tabular}{lcccccc}
\hline & $\begin{array}{c}\text { MPC-MDM } \\
(\%)\end{array}$ & $\begin{array}{c}\text { DH } \\
(\%)\end{array}$ & $\begin{array}{c}\text { GP } \\
(\%)\end{array}$ & $\begin{array}{c}\text { PD } \\
(\%)\end{array}$ & $\begin{array}{l}\text { WS } \\
(\%)\end{array}$ & $p$ value \\
\hline Without complications & 20.4 & 7.6 & 13.9 & 32.1 & 33.3 & $<0.001$ \\
Obstetrical complications & & & & & & \\
$\quad$ st trimester bleeding & 8.2 & 13.9 & 5.6 & 4 & 11.1 & 0.04 \\
PTD threat & 22.5 & 31.6 & 27.8 & 27 & 11.1 & 0.28 \\
Urinary track infections & 8.9 & 13.9 & 5.6 & 4.8 & 0 & 0.06 \\
Other infections & 20.2 & 5.1 & 0 & 2.4 & 0 & $<0.001$ \\
Anemia & 4.7 & 7.6 & 5.6 & 2.4 & 0 & 0.25 \\
Gestational hypertension & 4.1 & 1.3 & 5.6 & 3.2 & 0 & 0.62 \\
Preeclampsia & 4.7 & 2.5 & 11.1 & 2.8 & 11.1 & 0.13 \\
Hellp syndrome & 0.6 & 2.5 & 0 & 0 & 11.1 & 0.007 \\
Gestational diabetes & 6.4 & 2.5 & 0 & 5.6 & 0 & 0.29 \\
PROMPT & 17.1 & 43.6 & 27.8 & 21.1 & 22.2 & $<0.001$ \\
Fetal malformations & 2.3 & 0 & 2.8 & 0.8 & 0 & 0.36 \\
IUFD & 1.2 & 0 & 8.3 & 4 & 0 & 0.024 \\
TTTS & 1.7 & 5.1 & 2.8 & 2.4 & 0 & 0.37 \\
IUGR & 13 & 11.4 & 22.2 & 10.3 & 0 & 0.22 \\
Discordant growth & 7.2 & 7.6 & 0 & 4 & 0 & 0.15 \\
\hline
\end{tabular}

PTD - preterm delivery; PROMPT - preterm premature rupture of membranes; IUFD - intra uterine fetal death; TTTS - twin to twin transfusion syndrome; IUGR - intra uterine growth restriction. 
Table 2. Delivery data according to place of prenatal surveillance.

\begin{tabular}{|c|c|c|c|c|c|c|}
\hline & MPC-MDM & DH & GP & $\mathrm{PD}$ & WS & $p$ value \\
\hline \multicolumn{7}{|l|}{ Delivery type $(\%)$} \\
\hline Vaginal & 41.7 & 44.3 & 44.4 & 45.6 & 55.6 & 0.84 \\
\hline Cesarean & 13.8 & 8.9 & 11.1 & 11.1 & 11.1 & \\
\hline Cesarean to 2nd twin & 1.4 & 3.8 & 0 & 2.8 & 0 & \\
\hline Elective cesarean & 43 & 43 & 44.4 & 40.5 & 33.3 & \\
\hline \multicolumn{7}{|c|}{ Gestational age (GA) at delivery (\%) } \\
\hline$<28$ weeks & 1.4 & 24.4 & 8.3 & 7.1 & 25 & $<0.001$ \\
\hline 28-32 weeks & 11.4 & 48.7 & 30.6 & 16.7 & 12.5 & \\
\hline 33-34 weeks & 18.2 & 17.9 & 22.2 & 19.8 & 12.5 & \\
\hline $35-36$ weeks & 35.5 & 7.7 & 19.4 & 24.2 & 25 & \\
\hline$>36$ weeks & 33.5 & 1.3 & 19.4 & 32.1 & 25 & \\
\hline \multicolumn{7}{|c|}{ Birthweight Fetus1/Fetus2 (\%) } \\
\hline$<1000 \mathrm{~g}$ & $2.1 / 2.7$ & $26.6 / 27.8$ & $11.4 / 8.8$ & $6.8 / 7.6$ & $25 / 25$ & $<0.001$ \\
\hline $1000-1499 \mathrm{~g}$ & $6.2 / 5.8$ & $25.3 / 27.8$ & $14.3 / 17.6$ & $8 / 9.2$ & $0 / 12.5$ & \\
\hline $1500-1999 \mathrm{~g}$ & $13.9 / 19.6$ & $29.1 / 19$ & $28.6 / 35.3$ & $19.5 / 21.2$ & $12.5 / 0$ & \\
\hline $2000-2499 \mathrm{~g}$ & $43.2 / 36.9$ & $15.2 / 21.5$ & $22.9 / 20.6$ & $33.1 / 30.4$ & $25 / 25$ & \\
\hline$\geq 2500 \mathrm{~g}$ & $34.6 / 35$ & $3.8 / 3.8$ & $22.9 / 17.6$ & $32.7 / 31.6$ & $37.5 / 37.5$ & \\
\hline $\bar{M}$ ean \pm SD g & $2317 \pm 493$ & $1467 \pm 581$ & $1895 \pm 664$ & $2186 \pm 617$ & $1928 \pm 909$ & \\
\hline & $2280 \pm 535$ & $1467 \pm 610$ & $1871 \pm 647$ & $2134 \pm 631$ & $1841 \pm 905$ & \\
\hline \multicolumn{7}{|l|}{ NICU admission (\%) } \\
\hline Fetus 1 & 33.7 & 85.7 & 33.3 & 45.2 & 100 & $<0.001$ \\
\hline Fetus 2 & 37.2 & 85.2 & 33.3 & 42.5 & 100 & $<0.001$ \\
\hline F 1 and/or F 2 & 37.3 & 85.7 & 36.4 & 44.6 & 66.7 & $<0.001$ \\
\hline
\end{tabular}

GA <28: most frequently in cases WS or DH. with the lowest frequency observed in MPC-MDM.

GA28-32: more frequent in GP and DH and less frequent in MCP-MDM.

GA33-34: the surveillance sites did not differ.

GA35-36: most frequent in MPC-MDM and less frequent in DH and PD.

GA> 36: more frequent in MCP-MDM and less frequent in DH.

significantly higher rates of preterm birth $<28$ weeks in WS and $\mathrm{DH}$; and between 28 and 32 weeks, also significantly higher rates to DH and GP, with the lower rates in both intervals being in MPC-MDM women who also had the higher rates of deliveries at GA equal or superior to 35 weeks. The same was verified for birthweight, with higher rates of extremely low birthweight in women from WS and DH, and those from MPC-MDM with the lower rates. Regarding NICU admission, MPC-MDM women' fetuses had the significantly lower admission rates, unlike DH with the significantly higher admission rates.

We then evaluate whether the site of prenatal surveillance had significant influence on each of the outcomes mentioned through two logistic regression models, one to determine the predictors of pregnancy complications and another to determine the predictors of preterm delivery before 34 weeks.

In the first logistic regression, we found that obstetric complications occurrence is $52 \%$ less frequent in women with prenatal surveillance in MPC-MDM. We also verified that complications are also $2 \mathrm{x}$ higher in monochorionic twins Figure 1(a).

In the second logistic regression, we found that occurrence of preterm delivery before 34 weeks was $47.8 \%$ inferior in women with a prenatal surveillance in MPC-MDM, unlike those followed in DH with a risk $9.2 \%$ superior of having a delivery at that GA - Figure 1(b).

\section{Discussion}

This study contributes to this very important issue of prenatal surveillance of twin pregnancies, since there are no specific studies about this theme as a primary outcome. All we know is through the conclusions of many other studies about twins which state that for improving outcomes we must improve an accurate surveillance. It confirms the subjective impression that in referral centers which generally have strict protocols of surveillance, the outcomes are much better than the surveillance in other district hospitals or in the GP, were these women are followed may times like a singleton pregnant women.

Obstetrical complications like anemia, urinary track infections, preterm delivery threat, gestational hypertension, preeclampsia, gestational diabetes, fetal malformations, twinto-twin transfusion syndrome in monochorials, intrauterine discordant growth and intrauterine growth restriction reveal to be not significantly different among all types of prenatal surveillance. This fact may be due to the independency of these conditions to the level of surveillance applied. They are more likely to be dependent of women intrinsic conditions.

The prevalence of infections other then urinary significantly higher in MPC-MDM was surprising and may be explained by the more accurate diagnosis of vaginal infections due to the analysis of vaginal exudate by protocol once a trimester, and by the higher frequency of consultations that get women to complain of any other infectious symptom that lead to diagnosis. These facts, specially the higher detection of vaginal infection and its treatment, can also explain the lower frequency of preterm premature rupture of membranes in the MPC-MDM and higher in DH.

Intrauterine fetal death was also significantly less frequent in MPC-MDM women and more frequent in those from GP and PD, which can also be explain by the accuracy and frequency of the surveillance in a referral center, that lead to a more precocious detection of any complication or even slight 


\begin{tabular}{lccccc} 
(a) PREDICTORS & Beta & OR & IL CI95\% & SL CI95\% & p-Value \\
\hline MPC-MDM (versus all others) & $\mathbf{- 0 , 7 3 3}$ & $\mathbf{0 , 4 8 0}$ & $\mathbf{0 , 2 4 6}$ & $\mathbf{0 , 9 3 9}$ & $\mathbf{0 , 0 3 2}$ \\
Monochorial & 0,721 & 2,057 & 1,330 & 3,182 & 0,001
\end{tabular}
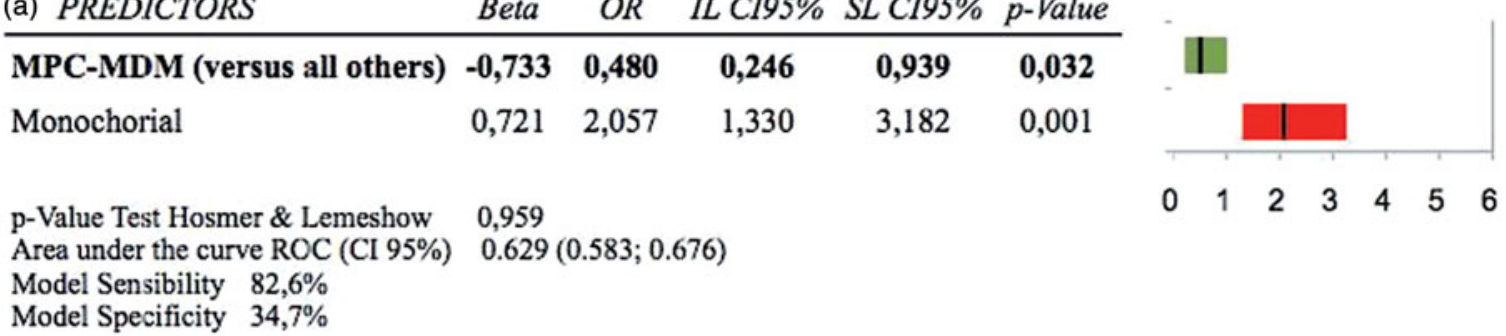

\begin{tabular}{lccccc} 
(b) $\quad$ PREDICTORS & Beta & OR & IL CI95\% & SL CI95\% & p-Value \\
\hline DH (vs all others) & $\mathbf{2 , 2 2 1}$ & $\mathbf{9 , 2 1 9}$ & $\mathbf{4 , 0 0 6}$ & $\mathbf{2 1 , 2 1 2}$ & $<\mathbf{0 . 0 0 1}$ \\
MPC-MDM (vs all others) & $\mathbf{- 0 , 6 4 9}$ & $\mathbf{0 , 5 2 2}$ & $\mathbf{0 , 3 7 9}$ & $\mathbf{0 , 7 2 1}$ & $<\mathbf{0 . 0 0 1}$
\end{tabular}

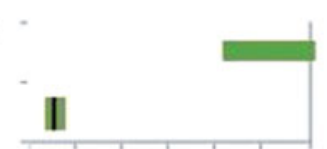

\author{
p-Value Test Hosmer \& Lemeshow \\ Area under the curve ROC (CI 95\%) \\ Model Sensibility $66,8 \%$ \\ Model Specificity $73,1 \%$
}

0,301

$0.765(0.732 ; 0.798)$

$\begin{array}{lllllll}0 & 1 & 2 & 3 & 4 & 5 & 6\end{array}$

Figure 1. (a) Logistic regression model to determine the predictors of pregnancy complications according to the prenatal surveillance place. (b) Logistic regression model to determine the predictors of preterm delivery at $\leq 34$ week according to the pernatal serveillance place.

alteration to normal evolution that promotes a visit in a shorter time to evaluated the fetal wellbeing.

No differences were found in cesarean rates, but gestational age at delivery, birthweights and admissions to NICU were significantly different. Again, considering all of these parameters, the prenatal surveillance in MPC-MDM lead to the better outcomes, with much lower rates of extremely and very low prematurity, of extremely and very low birthweight and of NICU admissions. These results are interconnected because the latter pregnancies terminate better chances of higher birthweight and lower risk of NICU admissions. These results are corroborated by one of the logistic regression model applied, which concluded that the occurrence of preterm delivery before 34 weeks was $47.8 \%$ inferior in women with a prenatal surveillance in MPC-MDM, unlike those followed in DH with a risk $9.2 \%$ superior of having a delivery at that GA.

The other logistic regression model used confirm our initial and subjective hypothesis that obstetric complications' occurrence is $52 \%$ less frequent in women with prenatal surveillance in MPC-MDM.

Given the lower complication rate, lower rate of prematurity and low birthweight with consequent lower rate of admissions to the NICU, our results demonstrate the crucial importance of prenatal surveillance to be carried out in a differentiated consultation in referral centers that have specific and strict protocols. Another solution is the urgent implementation of same protocols in all other places of surveillance, since we proved that this straight surveillance greatly reduces the occurrence of prenatal complications, mainly PROMPT, PTD and consequently all its neonatal morbidity and mortality associated.

\section{Declaration of interest}

The authors declare no conflict of interest.

\section{References}

1. American College of Obstetricians and Gynecologists Committee on Practice Bulletins-Obstetrics; Society for Maternal-Fetal Medicine; ACOG Joint Editorial Committee. ACOG Practice Bulletin \#56: Multiple gestation: complicated twin, triplet, and high-order multifetal pregnancy. Obstet Gynecol 2004;104:869-83.

2. Ferrarretti AP, Goossens V, de Mouzon J, et al. Assisted reproductive technology in Europe, 2008. Results generated from European registers by ESHRE. Human Reprod 2012;27:2571-84.

3. National Collaborating Centre for Women's and Children's Health (UK). Multiple pregnancy: the management of twin and triplet in the antenatal period. National Institute for Health and Clinical Excellence (NICE) Clinical Guidelines No. 129; September 2011. London: RCOG Press; 2011.

4. Graça LM. Gravidez múltipla. In: LIDEL, ed. Medicina maternofetal. 4th ed. Lisboa-Porto; 2010:465-80.

5. Büscher U, Horstkamp B, Wessel $J$, et al. Frequency and significance of preterm delivery in twin pregnancies. Int J Gynaecol Obstet 2000;69:1-7.

6. Lewi L, Gucciardo L, Van Mieghem T, et al. Monochorionic diamniotic twin pregnancies: natural history and risk stratification. Fetal Diagn Ther 2010;27:121-33. 